\title{
Thyroid Storm: Emergency Care for Thyrotoxicosis
}

Sam Hamseley.

Department of Medical sciences, Adama, Ethopia

\author{
*Corresponding Author : Sam Hamseley, Department of Medical sciences, Adama, Ethopia. E-mail: sam.hamseley@yahoo.co. in \\ Received date: April 10, 2018;Accepted date : April 20, 2018; Published date: May 10, 2018 \\ Citation for this Article : Sam Hamseley, Thyroid Storm: Emergency Care for Thyrotoxicosis J. Endocrinology and Disorders. \\ Doi: $10.31579 / 2640-1045 / 030$
}

Copyright : (c) 2018 Sam Hamseley.This is an open-access article distributed under the terms of the Creative Commons Attribution License, which permits unrestricted use, distribution, and reproduction in any medium, provided the original author and source are credited.

\section{Abstract}

Thyroid hormones affect all organ systems and, in excess, can cause increased metabolic rate, heart rate, ventricle contractility, and gastrointestinal motility as well as muscle and central nervous system excitability. Thyroid storm is the extreme manifestation of thyrotoxicosis with an estimated incidence of 0.20 per 100,000 per year among hospitalized patients in Japan. The mortality of thyroid storm without treatment ranges from $80 \%$ to $100 \%$; but with treatment, the mortality rate is between $10 \%$ and $50 \%$. The diagnostic strategy for thyroid storm may take into consideration Burch-Wartofsky scoring or Akamizu's diagnostic criteria. Multiple treatment aims need to be addressed in managing thyroid storm effectively. This paper puts together all aspects to be considered for the management of hyperthyroidism and thyroid storm during the acute and emergency phase as well as consideration of special populations.

\section{Keywords}

Acute; Emergency; Hyperthyroidism; Thyroid Storm; Thyrotoxicosis

\section{Introduction}

Thyroid storm (also known as thyroid or thyrotoxic crisis) is an uncommon condition reflecting an extreme physiological state within the spectrum of thyrotoxicosis. The condition is rare, however, mortality rates are high and may approach $10-20 \%$. Thyroid storm is most commonly seen in the context of underlying Graves' hyperthyroidism but can complicate thyrotoxicosis of any aetiology. Clinical features represent manifestations of organ decompensation, with fever seen almost universally.(1-5) Management is supportive with cooling and fluids, alongside measures taken to reduce thyroid hormone synthesis, hormone release and inhibition of the peripheral effects of excessive thyroid hormone. In addition, the management of thyroid storm should not disregard the search and appropriate treatment of any precipitating factors. (6-10).

\section{Pathophysiology}

Normal thyroid function is maintained by endocrine interactions between the hypothalamus, anterior pituitary and thyroid gland [Matfin, 2009]. Iodide is transported across the basement membrane of the thyroid cells by an intrinsic membrane protein called the $\mathrm{Na} / \mathrm{I}$ symporter (NIS). At the apical border, a second iodide transport protein called pendrin moves iodide into the colloid, where it is involved in hormono-genesis. Once inside the follicle, most of the iodide is oxidized by the enzyme thyroid peroxidase (TPO) in a reaction that facilitates combination with a tyrosine molecule to ultimately form thyroxine (T4) and triiodothyronine (T3). Thyroxine is the major thyroid hormone secreted into the circulation $(90 \%$, with $\mathrm{T} 3$ composing the other 10\%). There is evidence that T3 is the active form of the hormone and that $\mathrm{T} 4$ is converted into $\mathrm{T} 3$ before it can act physiologically.(11-16)

All of the major organs in the body are affected by altered levels of thyroid hormone. These actions are mainly mediated by T3. In the cell, T3 binds to a nuclear receptor, resulting in transcription of specific thyroid hormone response genes.(17-20).

\section{Aetiology}

Thyroid storm is most commonly associated with underlying Graves' disease, although has been reported with autonomous thyroid nodular disease. Traditionally, the condition was experienced frequently following thyroidectomy for thyrotoxic state (due to manipulation of the hyperactive thyroid gland during surgery), but modern treatments aimed at reducing preoperative thyroid output and hormone stores have dramatically reduced this complication.(21-25).

Regardless of the underlying aetiology of thyrotoxicosis, the rare transition to a state of thyroid storm usually requires a second superimposed insult. Most commonly this is infection, although trauma, surgery, myocardial infarction (MI), diabetic ketoacidosis (DKA), pregnancy and parturition have been reported as causes. The administration of large quantities of exogenous iodine (such as with iodinated contrast agents or amiodarone) can provide the substrate for significant thyroid hormone production and secretion if there are areas of autonomous thyroid tissue within the gland (i.e. Jod-Basedow phenomenon). Abrupt cessation of thionamide therapy (i.e. antithyroid drugs such as pro-pylthiouracil [PTU], methimazole and carbima-zole) usually due to poor patient adherence or other issues has been associated with worsening thyrotoxicosis and rarely descent into thyroid storm. Biological agents such as interleukin-2 and $\alpha$-interferon have been reported to induce thyroid storm when used to treat infectious disease, certain cancers and disorders of immune function.(26-32).

\section{Thyroid Storm Precipitation}

The most common precipitating cause of thyroid storm is infection. ${ }^{6}$ Other causes include diabetic ketoacidosis, hypoglycemia, hyperosmolar coma, pulmonary embolism, thyroid hormone overdose, withdrawal of antithyroid medications, iodinated contrast medium ingestion, vascular accidents, surgery, stress, parturition, eclampsia, trauma, and myocardial infarct. In some patients undergoing radioactive iodine therapy for hyperthyroidism, thyroid storm may ironically occur following treatment due to withdrawal of antithyroid drugs, release of thyroid hormones from damaged thyroid follicles, or the effect of radioactive iodine itself. In approximately $20-25 \%$ of cases, no acute precipitant is identified.(33-40). 


\section{Diagnostic considerations}

The diagnosis of thyroid storm must be made on the basis of suspicious but nonspecific clinical findings. If the diagnosis of thyroid storm is strongly suspected, waiting for the results of tests may cause a critical delay in the initiation of effective life-saving treatment. Furthermore, biochemical markers of thyroid function are not discernably different from thyrotoxic states without thyroid storm. Serum thyroid hormone levels (i.e. free T3 [FT3] and free T4 [FT4]) are elevated with suppressed TSH levels (with the rare exceptions being states of thyroid hormone resistance or TSH secreting pituitary adenomas) confirming the diagnosis of thyrotoxicosis.(41-45).

\section{Thyroid-specific therapy}

The immediate goals when treating thyroid storm are to decrease thyroid hormone synthesis, prevent thyroid hormone release, decrease peripheral action of circulating thyroid hormone to reduce heart rate and support the circulation, and to treat the precipitating condition [Nayak and Burman, 2006]. ( 46- 59)The therapeutic options for thyroid storm are the same as those for uncomplicated thyrotoxicosis, except that the drugs are given in higher doses and more frequently. When treating thyroid storm, one should consider the five 'Bs': Block synthesis (i.e. antithyroid drugs); Block release (i.e. iodine); Block T4 into T3 conversion (i.e. high-dose propylthiouracil [PTU], propranolol, corticosteroid and, rarely, amiodarone); Beta-blocker; and Block enterohepatic circulation (i.e. cholestyramine).

\section{Conclusions}

Thyroid storm is a rare endocrine emergency but is associated with high mortality. It most commonly occurs in the context of underlying Graves' thyrotoxicosis, but is frequently precipitated by a secondary event such as infection or MI. Prompt recognition of the condition with timely intervention is crucial, and management of the patient in an AMU, high-dependency or intensive care unit is essential. Treatment is based on immediate blockade of thyroid hormone synthesis, prevention of the release of further thyroid hormone from thyroid stores, and alleviation of the peripheral effects of thyroid hormone excess. A search for a precipitant for the thyroid storm is critical and should be treated promptly. Maintenance therapy takes into account disease-specific factors and patient preference, with measures taken to prevent a recurrence of thyroid storm.

\section{References}

1. Abraham P., Acharya S. (2010) Current and emerging treatment options for Graves' hyperthyroidism. Therapeut Clin Risk Managem 6: $29-40$.

2. Bahn R. (2010) Graves' opthalmopathy. N Engl J Med 362: 726-738

3. Burch H.B., Wartofsky L. (1993) Life-threatening thyrotoxicosis. Thyroid storm. Endocrinol Metab Clin North Am 22: 263 277https://doi.org/10.1016/S0889-8529(18)30165-8

4. Erdogan M.F., Gulec S., Tutar E., Baskal N., Erdogan G. (2003) A stepwise approach to the treatment of amiodarone-induced thyrotoxicosis. Thyroid 13: 205-209

5. Ezer A., Caliskan K., Parlakgumus A., Belli S., Kozanoglu I., Yildirim S. (2009) Preoperative therapeutic plasma exchange in patients with thyrotoxicosis. J Clin Apharesis 11: 111-114 https://doi.org/10.1089/105072503321319521

6. Food and Drug Administration (2010) Propylthiouracil tablets. (accessed 2 August 2010).https://doi.org/10.1002/cncr.25027

7. Kreisner E., Lutzky M., Gross J. (2010) Charcoal hemoperfusion in the treatment of levothyroxine intoxication. Thyroid 20: 209-212 [https://doi.org/10.1089/thy.2009.0054

8. Malozowski S., Chiesa A. (2010) Propylthiouracil-Induced Hepatotoxicity and Death. Hopefully, Never More. J. Clin. Endocrinol. Metab 95: 3161-3163https://doi.org/10.1210/jc.20101141

9. Matfin G. (2009) Disorders of endocrine control of growth and metabolism, In: Porth C.M., Matfin G., editors. (eds). Pathophysiology: Concepts of Altered Health States, 8th edn, Wolters Kluwer Health: Philadelphia, PA

10. Nayak B., Burman K. (2006) Thyrotoxicosis and thyroid storm. Endocrinol Metab Clin N Am 35: 663-686

11. D.A., Li J.C., Wei C.L., et al. (2005) The effect of combination therapy with propylthiouracil and cholestyramine in the treatment of Graves' hyperthyroidism. Clin Endocrinol (Oxf) 62: 521524.https://doi.org/10.1111/j.1365-2265.2005.02249.x 